Cahiers
de la Recherche
Fur les Droits
Fondamentaux

Cahiers de la recherche sur les droits

fondamentaux

$13 \mid 2015$

Le droit d'asile

\title{
La force des préjugés : regard critique sur les procédures accélérées
}

The Strength of Prejudice: A Critical Look at Fast-Track Procedures

\section{Christophe Pouly}

\section{(2) OpenEdition \\ Journals}

Édition électronique

URL : http://journals.openedition.org/crdf/1194

DOI : $10.4000 /$ crdf. 1194

ISSN : 2264-1246

Éditeur

Presses universitaires de Caen

Édition imprimée

Date de publication : 1 novembre 2015

Pagination : 65-75

ISBN : 978-2-84133-742-2

ISSN : $1634-8842$

Référence électronique

Christophe Pouly, «La force des préjugés : regard critique sur les procédures accélérées », Cahiers de la recherche sur les droits fondamentaux [En ligne], 13 | 2015, mis en ligne le 01 novembre 2016,

consulté le 12 février 2020. URL : http://journals.openedition.org/crdf/1194 ; DOI : 10.4000/crdf.1194

Cahiers de la recherche sur les droits fondamentaux 


\title{
La force des préjugés: regard critique sur les procédures accélérées
}

\author{
Christophe POULY \\ Docteur en droit public, avocat au barreau de Paris
}

\section{Le champ d'application des procédures accélérées}

A. Les demandes manifestement infondées en raison de considération extérieures au demandeur

B. Les demandes frauduleuses, abusives ou dilatoires

C. Les demandes ultérieures

\section{La mise en œuvre de la procédure accélérée}

A. L'instance compétente pour décider de la mise en œuvre de la procédure accélérée

B. Le maintien des principes de base et des garanties fondamentales

III. Le contrôle juridictionnel des procédures accélérées

IV. Le droit à un recours suspensif

Déterminer si un ressortissant d'un État tiers est fondé à obtenir le bénéfice d'une protection internationale reste sans doute l'une des tâches les plus délicates qui incombent désormais aux États membres de l'Union européenne, d'autant plus que l'Europe reste confrontée à une hausse constante des demandes de protection. Les politiques restrictives d'immigration légale combinées à la multiplication des conflits meurtriers à l'échelle mondiale ont fait de l'asile, comme le note Catherine Wihtol de Wenden, l'une des voies d'entrée légale dans les pays d'accueil ${ }^{1}$. De ce fait, au nombre des candidats à l'asile comptent des migrants dont l'exil est dicté pas des considérations économiques ou sociales exclusives des procédures de protection internationale mais qui n'ont trouvé que ce seul moyen pour tenter leur chance dans une Europe qui représente, à tort ou à raison, l'Eldorado. Plutôt que de reconsidérer leurs politiques d'immigration qui n'empêchent pas et ne pourront jamais empêcher les mouvements migratoires dictés aussi bien par des considérations économiques que par des considérations de sécurité, c'est en tout cas le constat que l'on peut tirer des dernières décennies, les États européens ont appréhendé ce phénomène sous l'angle exclusivement répressif. Séparer le bon grain de l'ivraie, détecter les faux demandeurs d'asile pour protéger les droits des "vrais» réfugiés, telle fut la réponse simple mais efficace pour faire baisser le nombre d'admissions au statut de réfugié et à la protection subsidiaire.

C'est dans ce contexte que la loi dicta de distinguer, parmi les demandeurs d'asile, ceux qui sollicitaient une protection de bonne foi de ceux « dont la demande repose sur une fraude délibérée ou constitue un recours abusif aux procédures d'asile ou n'est présentée qu'en vue de faire échec à une mesure d'éloignement prononcée ou imminente $»^{2}$. Demandeur-fraudeur, tel est le visage du

1. C. Wihtol de Wenden, La question migratoire au XXIe siècle: migrants, réfugiés et relations internationales, Paris, Presses de Sciences Po, 2013 , p. 26.

2. Art. 24 de la loi no 93-1027 du 24 août 1993 relative à la maîtrise de l'immigration, des conditions d'entrée, d'accueil et de séjour des étrangers en France, JORF, 29 août 1993, p. 12196. 
réfugié que nous renvoie la législation. Comme a pu l'écrire Jean-Michel Belorgey,

Qui ne voit en effet que l'enjeu de cet amalgame est d'assurer l'ajustement entre demande de protection pour de bon consentie, non en fonction de la légitimité des demandes, mais en fonction de l'idée que, à tort ou à raison, on se fait du possible ou du tolérable. Ce qu'on avoue naturellement jamais, c'est la légitimité des demandes qu'on met en doute ${ }^{3}$.

Selon l'auteur, la procédure de protection s'est alors transformée en course d'obstacles au terme de laquelle une infime partie des demandeurs en sort gagnant ${ }^{4}$.

Les deux premiers obstacles que doit franchir le demandeur d'asile sont la recevabilité et l'admission au séjour provisoire. Procédure dite normale, permettant de bénéficier de l'ensemble des garanties de procédure, de séjour et d'accueil $v s$ procédure accélérée, laquelle prive le demandeur de la plus grande partie de ces garanties. Selon le rapport établi par le gouvernement en application de l'article L. 111-10 du Code de l'entrée et du séjour des étrangers et du droit d'asile (CESEDA) pour l'année 2012, les procédures dites prioritaires ont concerné en France près de 14800 demandes d'asile, représentant près de $31,2 \%$ de la demande globale ${ }^{5}$. Ne sont pas prises en compte dans ces chiffres les demandes examinées en procédures à la frontière dont le caractère manifestement infondé fait l'objet d'un examen en procédure rapide qui, au sens du droit de l'Union, ne constitue pas une procédure accélérée ${ }^{6}$. Les placements en procédure prioritaire concernent principalement les ressortissants d'États tiers dits "pays d'origine sûrs", les demandes de réexamen ainsi que les demandes formulées en rétention administrative lesquelles, pour ces deux dernières catégories, sont généralement qualifiées de frauduleuses, de dilatoire ou d'abusive en tant qu'elles auraient pour but exclusif de faire échec à une mesure d'éloignement. Ils sont également mis en œuvre lorsque les demandeurs ne font parvenir leur dossier dûment complété à l'Office français de protection des réfugiés et apatrides (OFPRA) qu'au-delà de la date butoir fixée à vingt et un jours après la remise du formulaire, quelle qu'en soit la raison. L'usage immodéré du recours à la procédure prioritaire en France a été souvent critiqué, tant par les organes internes tels que la Commission nationale consultative des droits de l'homme $(\mathrm{CNCDH})^{7}$, le législateur lui-même ${ }^{8}$, et a surtout fait l'objet d'une condamnation par la Cour européenne des droits de l'homme (Cour EDH) 9 .
Le droit de l'Union encadre désormais le régime procédural de l'examen des demandes de protection. La directive $n^{\circ}$ 2013/32/UE du Parlement européen et du Conseil du 26 juin 2013 relative à des procédures communes pour l'octroi et le retrait de la protection internationale, dite directive "Procédures ${ }^{10}$, fixe de manière précise les conditions de mise en œuvre des procédures accélérées et des procédures prioritaires. Au sens de la directive, la procédure prioritaire tend à raccourcir la durée globale de la procédure lorsque le demandeur est considéré comme étant une personne vulnérable ou que la demande est probablement fondée. La célérité est donc exigée dans l'intérêt du demandeur. En revanche, la procédure accélérée vise à évacuer les demandes qui, en fonction de circonstances précises que nous évoquerons par la suite, peuvent apparaître comme étant vouées à l'échec. L'exigence de célérité est alors dans l'intérêt de l'État membre. Ainsi le droit de l'Union crée d'une certaine façon trois catégories de demandes de protection bénéficiant pour chacune d'entre elles d'un régime juridique spécifique quant aux délais d'instruction et aux droits afférents: procédures prioritaires pour les demandes manifestement fondées ou émanant de personnes vulnérables, procédures accélérées pour les demandes manifestement infondées et, enfin, une procédure de droit commun, que l'on peut qualifier de "procédure normale » pour toutes les autres.

Le gouvernement français a dû, par voie de conséquence, programmer une réforme du droit d'asile afin d'ajuster la législation à cette structure. La procédure accélérée, qui se substitue à la procédure prioritaire, fait l'objet d'une transposition assez proche de la directive, bien qu'il ne s'agisse que d'une clause optionnelle et que les États membres peuvent prévoir des dispositions plus favorables. Tel n'a pas été le choix des autorités françaises qui, outre le fait de transposer minutieusement les dispositions les plus répressives, les a soumises à un régime procédural dont on peut douter, cette fois-ci, qu'il est bien conforme à l'esprit de la directive lorsque les demandeurs sont placés en rétention administrative.

Les logiques financières qui structurent désormais l'action administrative ont également une incidence directe sur la gestion technique des demandes de protection. Pour les gestionnaires de l'État, au terme de raisonnements qui empruntent parfois quelques raccourcis conceptuels, la rapidité est devenue synonyme de garantie des droits. L'action des organes de protection est encadrée par un «contrat d'objectifs et de performances » visant à réduire les délais d'examen des demandes d'asile (3 mois

3. J.-M. Belorgey, Le droit d'asile, Paris, LGDJ, 2013, p. 17.

4. Ibid.

5. Les étrangers en France, année 2012, dixième rapport établi en application de l'article L. 111-10 du CESEDA, p. 104.

6. Raison pour laquelle les procédures à la frontière ne seront pas abordées dans le cadre de la présente étude.

7. CNCDH, Les conditions d'exercice du droit d'asile en France, Paris, La documentation française, 2006, p. 69.

8. J.-Y. Leconte, C.-A. Frassa, Rapport d'information fait au nom de la commission des lois, $\mathrm{n}^{\circ}$ 130, enregistré à la présidence du Sénat le 14 novembre 2012, p. 27.

9. Cour EDH, 2 février 2012, I. M. c. France, nº 9152/o9.

10. Cette directive constitue la refonte de la directive $n^{\circ} 2005 / 85 / C E$ du Conseil du $1^{\text {er }}$ décembre 2005 relative à des normes minimales concernant la procédure d'octroi et de retrait du statut de réfugiés dans les États membres, appelée aussi directive "Procédures». 
pour l'OFPRA, 6 mois pour la Cour nationale du droit d'asile (CNDA) $)^{11}$. Ainsi, parallèlement aux procédures accélérées et aux procédures prioritaires, les procédures de droit commun deviennent aussi des procédures que l'on pourrait qualifier de rapides. Pourtant, l'exigence de rendement n'est pas compatible avec un examen minutieux des demandes de protection qui impose d'abord aux demandeurs de réunir des preuves de leurs allégations, preuves difficiles et longues à obtenir, qui impose un, voire plusieurs, entretiens avec un officier de protection, lequel devra procéder à de laborieuses recherches pour connaître le contexte géopolitique dans lequel s'inscrivent les faits allégués. La directive "Procédures» laisse aux États une marge d'appréciation quant à la fixation des délais d'examen des demandes, tant en première instance que sur les recours, ce délai devant être "raisonnable $»^{12}$. Le rapport de la Commission européenne relatif à l'application de la directive «Procédures» fait état d'une profonde disparité. Ainsi, le délai imparti à l'autorité responsable pour statuer sur la demande varie entre 48 heures (Lituanie), 3 jours (Bulgarie, Malte, Roumanie et Royaume-Uni), 4 jours (procédures de rétention en Espagne et en France), 10 jours (Chypre), 15 jours (France et procédures de rétention en Belgique), 1 mois (Grèce, République tchèque et Pologne), 2 mois (République slovaque, Luxembourg et Belgique) et 3 mois (Autriche et Espagne) ${ }^{13}$.

La transposition de la directive « Procédures» en droit interne aurait pu participer d'une élévation de degré de protection des demandeurs contre les aléas politiques et administratifs auxquels leurs demandes sont soumises. Nous verrons toutefois que tant par l'extension de leurs champs d'application que par le régime procédural commun, les procédures accélérées accentuent la précarité juridique des demandeurs d'asile et affectent, en dernière analyse, la qualité de l'examen de leur demande.

\section{Le champ d'application des procédures accélérées}

La mise en œuvre d'une procédure accélérée repose sur le postulat que la demande sera vouée probablement à l'échec ou que, à tout le moins, il n'est pas utile de mobiliser les moyens habituels pour statuer sur une demande dont il est presque préjugé qu'elle sera rejetée. Comme le soulignait l'avocat général Pedro dans ses conclusions sur l'arrêt Samba Diouf, dans certains cas, la procédure accélérée «constitue en réalité une procédure de refus anticipée ${ }^{14}$.
Afin de limiter les risques d'arbitraire, le législateur européen a tenté de définir, de façon plus ou moins précise, des critères objectifs autorisant les États membres à y recourir. On peut distinguer deux grandes catégories de motifs : les demandes manifestement infondées (A) et les demandes frauduleuses, dilatoires ou abusives (B). Les demandes de réexamen, dites demandes ultérieures, répondent parfois à l'une ou l'autre de ces catégories (C).

\section{A. Les demandes manifestement infondées en raison de considération extérieures au demandeur}

Il s'agit en premier lieu des demandes émanant de ressortissants provenant d'un pays d'origine sûr ${ }^{15}$. Le principe de la mise en œuvre de la procédure accélérée fondé sur le critère de la nationalité ou du pays d'origine a été validé par la Cour de justice de l'Union européenne (CJUE) ${ }^{16}$. Lors de la préparation de la refonte de la directive «Procédures », il fut envisagé la création d'une liste européenne. Cette option fut finalement abandonnée en raison des divergences persistantes entre les États membres, ce qui n'est pas sans poser un véritable problème au regard de l'exigence d'un régime d'asile européen commun. Il appartient donc aux États membres d'établir une liste de pays d'origine jugés sûrs, en s'appuyant sur diverses sources d'informations émanant du Haut-Commissariat pour les réfugiés des Nations unies (HCR), du Bureau européen d'appui en matière d'asile, du Conseil de l'Europe ou de toute autre organisation internationale ${ }^{17}$. La compétence propre des États membres dans l'élaboration de cette liste affecte nécessairement l'harmonisation du droit et des pratiques en matière d'asile. Comme le note $\mathrm{M}^{\mathrm{me}}$ Chantal Guittet, dans son avis sur la réforme de l'asile,

[...] certains pays européens n'ont pas adopté une telle liste au plan national, quand d'autres en sont dotés: les listes elles-mêmes sont très variables, à commencer par le nombre de pays inscrits ${ }^{18}$.

C'est pourquoi la CNCDH a considéré, pour sa part, que la directive «Procédures» n'apportait pas de garanties "pour désigner avec certitude les pays d'origine sûrs [...] et donner un minimum de cohérence attendue dans le cadre d'un régime d'asile européen ${ }^{19}$.

En droit français, l'article L. 741-4-2 ${ }^{\circ}$ du CESEDA définit le pays d'origine sûr comme étant celui dans lequel les principes de la liberté, de la démocratie et de l'état

11. Mission Immigration, asile et intégration, projet annuel de performance, annexe au projet de loi de finances pour 2015 , p. 19.

12. Art. $21, \$ 9$, de la directive $2013 / 32 / \mathrm{UE}$.

13. $\operatorname{COM}(2010) 465$ final, p. 15 .

14. C. V. Pedro, conclusions sur CJUE, $2^{\mathrm{e}}$ ch., 28 juillet 2011, Brahim Samba Diouf c. ministre du Travail, de l'Emploi et de l'Immigration, aff. C-69/10.

15. Art. $31, \S 8$, sous a, de la directive $2013 / 32 / \mathrm{UE}$.

16. CJUE, $2^{\mathrm{e}}$ ch., 31 janvier 2013 , H. I. D. \& B. A. c. Refugee Applications Commissioner (Ireland), aff. C-175/11.

17. Art. 37 de la directive $2013 / 32 / \mathrm{UE}$.

18. C. Guittet, Avis présenté au nom de la commission des affaires étrangères sur le projet de loi relatif à la réforme de l'asile, $\mathrm{n}^{\circ} 2357$, enregistré à la présidence de l'Assemblée nationale le 12 novembre 2014, p. 31.

19. $\mathrm{CNCDH}$, Ass. plén., Avis sur le projet de loi relatif à la réforme de l'asile, 20 novembre 2014, p. 12. 
de droit, ainsi que des droits de l'homme et des libertés fondamentales sont respectés. Le projet de loi relatif à la réforme de l'asile prévoit une définition plus précise:

[...] lorsque, sur la base de la situation légale, de l'application du droit dans le cadre d'un régime démocratique et des circonstances politiques générales, il peut être démontré que, d'une manière générale et uniformément, il n’y est jamais recouru à la persécution ni à la torture ni à des peines ou traitements inhumains ou dégradants et qu'il n'y a pas de menace en raison d'une violence aveugle dans des situations de conflit armé international ou interne ${ }^{20}$.

Périodiquement, le conseil d'administration de l'OFPRA révise cette liste, au gré des aléas géopolitiques.

Toutefois, selon l'article $36, \$ 1$, de la directive «Procédures ", un pays d'origine sûr ne peut être considéré comme tel pour un demandeur que si celui-ci en est originaire ou, apatride, en était résident, et si ce demandeur n'a pas fait valoir de raisons sérieuses permettant de penser qu'il ne s'agit pas d'un pays d'origine sûr en raison de sa situation personnelle. Autrement dit, la seule circonstance que le demandeur provienne d'un pays tiers dit sûr ne suffit pas, à elle seule, à autoriser la mise en ouvre d'une procédure accélérée. Il appartiendrait à l'autorité responsable d'apprécier si, de prime abord, par une sorte de pré-jugement, la situation dans laquelle aurait été placé le demandeur contredirait le caractère sûr du pays d'origine.

En second lieu, les procédures accélérées sanctionnent également des demandes de protection manifestement infondées en raison de motifs sans pertinence au regard des critères d'attribution d'une protection internationale.

Il s'agit en fait d'exclure de la procédure normale toutes les demandes de protection justifiées par des raisons étrangères aux critères d'attribution de la qualité de réfugié ou de la protection subsidiaire, telles que les demandes fondées sur des considérations familiales ou économiques. La pertinence des questions soulevées, au sens de la directive, doit donc se rattacher directement aux critères de qualification des motifs de protection. À ce stade, il ne s'agit donc pas de savoir si les faits allégués sont établis mais seulement s'ils se rattachent à un motif de protection.

\section{B. Les demandes frauduleuses, abusives ou dilatoires}

D’une part, les procédures accélérées visent surtout les demandes frauduleuses dans le sens où le demandeur ferait des déclarations manifestement incohérentes et contradictoires, manifestement fausses ou peu plausibles qui contredisent des informations suffisamment vérifiées du pays d'origine, ce qui rend sa demande visiblement peu convaincante quant à sa qualité de bénéficiaire d'une protection internationale ${ }^{21}$.

L'autorité responsable apprécie, a priori, la qualité des allégations quant à leur cohérence et, d'une certaine façon, quant à leur caractère manifestement crédible. Cette prérogative reste toutefois délicate à mettre en œuvre. D'abord parce que les demandes de protection sont parfois formulées très maladroitement en raison notamment des traumatismes subis ou des difficultés à restituer, par un récit construit, le déroulement d'événements parfois dramatiques. Les récits incohérents ou contradictoires ne signifient pas nécessairement qu'ils sont faux. Bien au contraire, l'état de confusion dans lequel se trouve une victime ou un témoin de faits traumatisants conduit ces derniers à reconstruire, de manière désordonnée, le fil des événements. C'est pourquoi au stade du premier examen d'une demande de protection, la confusion des propos ne devrait pas conduire à privilégier une procédure accélérée qui, d'une certaine façon, vient priver le demandeur du temps qui lui est nécessaire pour réagencer les fragments de sa mémoire.

D’autre part, les procédures accélérées sanctionnent l'usage de faux documents d'identité ou de voyage, ou le fait de mentir. Dans sa note sur le projet de loi relatif à l'asile, le HCR a souligné que «l'absence de documents ou l'utilisation de faux documents ne rend pas en soi la demande frauduleuse, ni ne permet de tirer des conclusions négatives sur la sincérité de la demande $»^{22}$. Il est évident que la crainte qu'exprime l'organisation internationale, qui résulte notamment de ses observations, est que la procédure accélérée soit mise en œuvre de manière systématique dès qu'un demandeur aura utilisé, à quelque moment que ce soit, des faux documents. Ainsi, comme le rappelle le Haut-Commissariat,

Les demandeurs d'asile sont fréquemment obligés de fuir par des moyens irréguliers. Ils peuvent, de surcroît, avoir détruit ou s'être défait de pièces d'identité ou de titre de voyage, après y avoir été contraints par des passeurs ${ }^{23}$.

La dissimulation d'information importante peut être également guidée par la crainte d'être renvoyé dans son pays d'origine, parfois même dans un pays de l'Union européenne. L'affaire M. S. S. c. Belgique et Grèce a mis en lumière les mauvais traitements auxquels les demandeurs d'asile étaient exposés ${ }^{24}$ conduisant ces derniers à dissimuler certaines informations essentielles dans leurs parcours.

Mais la question de l'identité se pose de manière récurrente sous l'angle des dysfonctionnements dans

20. Projet de loi relatif à la réforme de l'asile, $\mathrm{n}^{\circ}$ 2182, enregistré à la présidence de l'Assemblée nationale le 23 juillet 2014, art. 6, en ligne: http:// www.assemblee-nationale.fr/14/projets/pl2182.asp

21. Art. $31, \$ 8$, sous e, de la directive 2013/32/UE.

22. HCR, Note du HCR sur le projet de loi relatif à la réforme de l'asile, octobre 2014, p. 7.

23. Ibid.

24. Cour EDH, GC, 21 janvier 2011, M. S. S. c. Belgique et Grèce, nº 30696/o9. 
l'enregistrement, par les autorités des États membres, des demandes d'asile. Une traduction défectueuse, une transcription d'un nom par exemple pachtoune, perse ou arabe en phonétique slave, germanique ou celtique peut réserver quelques surprises. Un nom d'épouse ici, puis le nom de jeune fille là, l'aléa de la conversion de la date de naissance, parfois même à peine connue, du calendrier musulman au calendrier grégorien, sont autant de sources d'erreurs de transcription qui sont ensuite qualifiées de «fausses identités». Or, il n'est pas rare que les différences dans l'orthographe d'un nom, ou dans le fait que les éléments patronymiques ne soient pas complets, sont analysées, de manière presque systématique, comme résultant d'une volonté de déclarer des identités différentes.

Dans ces deux hypothèses, le projet de loi relatif à la réforme de l'asile conduit l'OFPRA à procéder à un premier examen du caractère manifestement infondé de la demande. Si, après un examen plus minutieux, l'agent de protection estime que cette demande s'avère mieux fondée, il devrait substituer la procédure normale à la procédure accélérée. Mais on peut s'interroger sur la pertinence du mécanisme qui, en fait, permet d'analyser le sérieux de la demande à un stade avancé de son examen. Comme le soulignait le HCR, indépendamment du fait que l'erreur de qualification n'est susceptible d'aucune sanction dès lors que la CNDA exerce un contrôle de plein contentieux, il est probable que l'OFPRA s'appuie sur les mêmes motifs pour rejeter la demande au fond.

Enfin, les procédures accélérées sanctionnent le comportement du demandeur. Il s'agit d'abord de décourager ceux qui refuseraient de se soumettre au relevé de leurs empreintes digitales. Celles-ci sont en effet prises systématiquement afin de procéder à la détermination de l'État responsable de l'examen de la demande de protection. Refuser la prise d'empreintes fait obstacle à l'État saisi d'une demande de procéder à la détermination de l'État responsable. Le Conseil d'État a validé, dans un premier temps, les refus d'enregistrement des demandes d'asile aux motifs que l'impossibilité d'exploiter les empreintes, circonstance imputée au demandeur, empêchait l'instruction de la demande de protection ${ }^{25}$. Toutefois, le Conseil d'État est ensuite revenu sur une position plus mesurée en estimant que le directeur de l'OFPRA ne pouvait donner pour instruction de rejeter systématiquement les demandes déposées par des requérants dont les empreintes étaient inexploitables en écartant par ailleurs toute possibilité d'audition, cette circonstance ne pouvant faire obstacle à un examen individuel des demandes d'asile ${ }^{26}$. Sur ce point, la directive «Procédures» ne permet plus à l'autorité administrative de refuser l'enregistrement de la demande.

La procédure accélérée vise également le ressortissant d'un État tiers qui, entré ou ayant séjourné illégalement sur le territoire d'un État, n'aura pas présenté de demande de protection dans des délais brefs, sans motif valable.
La question est alors de savoir, d'une part, comment l'intéressé pourra déterminer la date de son entrée sur le territoire si celle-ci s'est faite irrégulièrement et, d'autre part, ce que l'on peut considérer comme étant un délai bref.

Le projet de loi relatif à la réforme de l'asile fixait ce délai à 90 jours. L'Assemblée nationale a amendé le texte en le portant à 120 jours. Mais dans tous les cas, il faudra que le demandeur rapporte la preuve de sa date d'entrée en France pour contrôler le respect de ce délai. Or, à l'exception de l'entrée par voie aérienne sous couvert d'un visa, les demandeurs d'asile arrivent en France soit par voie aérienne sans justifier de visa, cas dans lequel leur demande est examinée à la frontière selon une procédure spéciale, soit par voie terrestre, ce qui implique la mise en œuvre du règlement Dublin, rendant leur demande de protection irrecevable. Autrement dit, l'obligation de justifier de la date d'entrée sur le territoire a pour effet indirect le placement en procédure accélérée de l'ensemble des demandeurs arrivés par voie terrestre qui n'auraient pas été signalés par les États membres qu'ils auraient traversés car, en présentant des documents susceptibles d'établir qu'ils étaient sur le territoire d'un autre État membre moins de 120 jours auparavant $^{27}$, ce qui permet d'en déduire une présence récente, ils conduisent l'État sur le territoire duquel ils déposent leur demande à mettre en œuvre la procédure Dublin, de tels documents constituant des preuves et indices du franchissement irrégulier d'une frontière extérieure d'un autre État membre au sens des articles 13 et 22 du règlement Dublin. L'exigence de justifier de la date d'entrée sur le territoire présente un risque certain car, faute de pouvoir rapporter la preuve de l'entrée, un demandeur d'asile est toujours présumé déposer sa demande tardivement. En tout cas, dans le cadre d'un recours contentieux, en cas de grief tiré du caractère tardif du dépôt d'une demande, il appartient au requérant de rapporter la preuve contraire de ce qui lui est opposé.

La procédure accélérée vise également les demandeurs qui introduiraient une demande de protection dans le seul but de faire échec à l'exécution d'une mesure d'éloignement, demande que la loi qualifie également de dilatoire. Pour autant, une demande d'asile déposée dans un temps voisin d'une mesure d'éloignement ne signifie pas nécessairement qu'elle tend exclusivement à faire échec à l'exécution de cette mesure. Elle vise à l'empêcher, c'est évident, puisque l'intéressé redoute d'être renvoyé dans son pays d'origine. La question est de savoir si l'existence d'une mesure d'éloignement crée une présomption irréfragable de mauvaise foi. En droit, non, en fait, oui. Et c'est précisément ce qui fut l'objet de la condamnation de la France par la Cour européenne des droits de l'homme dans l'affaire I. M. c. France qui releva alors le caractère automatique du placement en procédure dite prioritaire lorsque les intéressés déposent leur demande de protection en rétention administrative. 
Enfin, les ressortissants d'États tiers dont la présence constitue une menace grave pour l'ordre public relèvent également de la procédure accélérée. Plus classique, en tant qu'elle se rattache au pouvoir de police, la réserve d'ordre public est en fait moins fréquemment utilisée.

\section{Les demandes ultérieures}

Les demandes ultérieures (ou demandes de réexamen) peuvent également impliquer un traitement en procédure accélérée. Ces demandes peuvent être classées en deux grandes catégories: les demandes irrecevables qui n'appellent pas d'examen sur le fond et les demandes recevables.

Une demande de réexamen doit faire l'objet d'un examen préliminaire, afin de déterminer si elle est justifiée par des éléments ou faits nouveaux. Le demandeur devra justifier des raisons pour lesquelles il a été dans l'impossibilité de faire valoir ces faits à l'occasion de l'examen de sa demande initiale ${ }^{28}$. Si les éléments ne sont pas suffisamment convaincants, une décision d'irrecevabilité sera opposée et la procédure sera clôturée.

Si la demande est jugée recevable, son examen relève du régime des procédures accélérées. C'est d'ailleurs en ce sens que le gouvernement français a interprété ces dispositions. L'article 7 du projet de loi dispose en effet que: «L'office statue en procédure accélérée lorsque [...] $2^{\circ}$ le demandeur a présenté une demande de réexamen qui n'est pas irrecevable». Il s'agit donc d'une procédure accélérée de plein droit, sous réserve que l'OFPRA décide, au cours de l'examen de la demande, de ne plus statuer en procédure accélérée lorsque cela lui paraît nécessaire pour procéder à un examen approprié de la demande ${ }^{29}$.

\section{La mise en œuvre de la procédure accélérée}

La spécificité des procédures accélérées, au stade de leur instruction en première instance, pose deux séries de questions. D'une part, quelle est l'autorité qualifiée pour décider de sa mise en œuvre (A) et, d'autre part, dans quelle mesure affectent-elles la substance des garanties fondamentales (B)?

\section{A. L'instance compétente pour décider de la mise en œuvre de la procédure accélérée}

Les procédures accélérées sont diligentées sans préjudice des principes de base et des garanties fondamentales. Bien que l'article 4 de la directive ne figure pas aux chapitres de ces principes, le considérant 17 précise que:

Pour garantir que les demandes de protection internationale soient examinées et que les décisions les concernant soient prises de manière objective et impartiale, il est nécessaire que les professionnels agissant dans le cadre des procédures prévues par la présente directive exercent leurs activités dans le respect des principes déontologiques applicables ${ }^{30}$.

Plus qu'un principe essentiel, le droit à un examen approprié d'une demande de protection constitue la clé de voûte du système de protection.

La directive «Procédures » impose aux États membres de désigner «pour toutes les procédures une autorité responsable de la détermination qui sera chargée de procéder à un examen approprié des demandes conformément à la présente directive ${ }^{31}$. Cette autorité doit disposer d'un personnel «compétent» et "dûment formé» afin de procéder à un examen approprié des demandes, que ce soit au stade de l'examen de la recevabilité, de l'examen au fond ou, le cas échéant, de la pertinence de la mise en œuvre de la procédure accélérée.

Le droit français, à cet égard, n'est pas tout à fait conforme au droit de l'Union. En effet, si l'OFPRA peut être regardée comme étant l'autorité responsable de l'examen des demandes de protection, le placement en procédure accélérée relève de la compétence du préfet. Le projet de loi relatif à la réforme de l'asile ne remet pas en cause cette répartition des compétences même s'il est prévu que l'OFPRA puisse de sa propre initiative, dans certains cas, décider du régime de la procédure et, dans tous les cas, reclasser en procédure normale une procédure antérieurement diligentée par le préfet sous le régime accéléré. En effet, le projet de loi prévoit, comme nous l'avons vu précédemment, des procédures accélérées de plein droit, celles relevant d'une décision de l'OFPRA et celles relevant d'une décision de l'autorité administrative. Pour le HCR, une telle dualité de compétence ne répond pas à l'objectif poursuivi par la directive qui est d'améliorer la qualité des décisions prises par une seule et même autorité en première instance et dont l'article 4 n'a pas limité la responsabilité au seul examen au fond ${ }^{32}$. En outre, selon le HCR, les préfectures ont une interprétation divergente des dispositions relatives à la procédure dite prioritaire. C'est pourquoi, seule une compétence unifiée serait de nature à garantir une application uniforme des procédures accélérées par des personnels parfaitement compétents ${ }^{33}$.

Une première tentative de transfert de compétence a été tentée par le ministre de l'Intérieur suite à la condam-

28. Art. 40, $\$ 4$, de la directive 2013/32/UE.

29. Projet de loi relatif à la réforme de l'asile, art. 7.

30. Cons. 17 de la directive 2013/32/UE.

31. Art. $4, \$ 1$, de la directive $2013 / 32 / \mathrm{UE}$.

32. HCR, Note du HCR sur le projet de loi relatif à la réforme de l'asile.

33. Ibid. 
nation de la France par la Cour européenne des droits de l'homme dans l'affaire I. M. c. France, dans le cas de demandes déposées en rétention administrative. Une note du 5 décembre $2013^{34}$ invitait les préfets à statuer sur le caractère prioritaire après avis du directeur de l'OFPRA. Cette note a été annulée par le Conseil d'État au motif que le ministre de l'Intérieur ne pouvait légalement charger le directeur de l'OFPRA de décider du régime de la procédure, l'établissement n'étant pas placé sous son autorité ${ }^{35}$. Dans l'attente du vote de la loi réformant la procédure d'asile, le ministre de l'Intérieur est revenu à une solution plus orthodoxe, au regard de la légalité, mais peu conforme au droit de l'Union. Il appartient à nouveau aux préfets d'exercer leur compétence sur le choix de la procédure en appréciant, au cas par cas, les circonstances ayant conduit un ressortissant d'un État tiers à déposer une demande de protection en rétention ${ }^{36}$. Pour apprécier le caractère dilatoire de la demande, le préfet doit prendre en considération la circonstance qu'il s'agit d'une première demande ou d'une demande de réexamen et surtout les raisons pour lesquelles cette demande n'a été déposée qu'après un placement en rétention administrative. Il est toutefois peu probable que l'autorité administrative qui a pris la mesure d'éloignement et qui a procédé au placement en rétention permette au ressortissant d'État tiers de recouvrer la liberté au seul motif qu'il aurait déposé une demande d'asile, quand bien même l'intéressé aurait à faire valoir des circonstances de nature à établir le bien-fondé de cette demande. En outre, le système transitoire conçu par le ministre de l'Intérieur ne permet pas au préfet de vérifier l'existence ou la pertinence d'éléments ou de faits nouveaux susceptibles d'appuyer le bien-fondé de cette demande en raison du principe de confidentialité des demandes d'asile faisant obstacle à ce que les éléments de fait, destinés exclusivement à l'OFPRA, soient portés à la connaissance de l'autorité administrative.

En dernière analyse, la fonction de police qui échoit aux services préfectoraux n'est pas compatible avec l'exercice d'une liberté fondamentale qu'est le droit d'asile. La pratique montre en effet que l'action administrative est trop souvent porteuse de restrictions, voire de violations, des garanties fondamentales. L'abondante jurisprudence des juges administratifs des référés en témoigne. La seule réserve de compétence que l'on peut reconnaître à l'autorité administrative pourrait se rapporter à la sauvegarde de l'ordre public, dont il lui appartient d'en assurer le maintien.

\section{B. Le maintien des principes de base et des garanties fondamentales}

Le placement en procédure accélérée ne peut pas priver le demandeur des garanties fondamentales liées à sa demande de protection. L'article 31, $\$ 8$, de la directive «Procédures» autorise les États à recourir aux procédures accélérées sous réserve du respect des principes de base et des garanties fondamentales prévues par le droit de l'Union.

Il s'agit d'abord du droit de rester sur le territoire de l'État membre, au cours de l'examen en première instance. Droit qui se rattache plus ou moins à la protection contre le refoulement garanti par l'article 33 de la Convention de Genève relative à la protection des réfugiés ${ }^{37}$. En droit français, le placement en procédure prioritaire est la conséquence du refus d'admettre le demandeur au séjour provisoire. Le demandeur ne pouvait alors être éloigné avant que l'OFPRA n'ait pris sa décision, sans pour autant être autorisé à résider provisoirement sur le territoire. Comme l'avait justement souligné Catherine Teitgen-Colly, le législateur avait créé « une sorte de droit au séjour irrégulier ${ }^{38}$.

L'article 9, $\$ 1$, de la directive «Procédures» précise in fine que le droit de rester dans l'État membre ne constitue pas un droit à un titre de séjour, elle ne fait en revanche aucune distinction entre procédure normale et procédure accélérée sur ce point. Seuls sont exclus du droit au séjour les demandeurs qui formulent des demandes dites ultérieures, au sens de l'article 41 de la directive, déposées dans le but de retarder ou d'empêcher l'exécution d'une mesure d'éloignement ou après le rejet d'une précédente demande ultérieure irrecevable ou une décision finale considérant la demande de protection comme étant manifestement infondée ${ }^{39}$, ou les demandeurs qui font l'objet d'une procédure d'extradition en exécution d'un mandat d'arrêt européen ou vers un État tiers ou une juridiction pénale internationale. C'est pourquoi, a priori, les demandeurs relevant de la procédure accélérée devraient bénéficier de la remise d'un document provisoire de séjour au cours de la procédure.

Il s'agit en second lieu des garanties procédurales telles que le droit à bénéficier de l'ensemble des informations dans une langue comprise, le droit à l'assistance d'un interprète, la possibilité de communiquer avec le HCR, le droit à l'assistance d'un conseil et enfin d'avoir communication des principaux éléments de la

34. Ministère de l'Intérieur, note d'information, 5 décembre 2013, NOR: INTV1327386N, BO du ministère de l'Intérieur, nº $2014-01,15$ janvier 2014.

35. CE, 30 juillet 2014, Cimade, $\mathrm{n}^{\circ} 375430$.

36. Ministère de l'Intérieur, information, 23 décembre 2014, NOR: INTV1430936N.

37. Convention de Genève du 28 juillet 1951 relative au statut des réfugiés, art. 33.

38. C. Teitgen-Colly, «Le droit d'asile: la fin des illusions», L'actualité juridique. Droit administratif, nº 2, février 1994, p. 97.

39. L'article 2, sous q, définit la "demande ultérieure» comme étant une nouvelle demande de protection internationale présentée après qu'une décision finale a été prise sur une demande antérieure, y compris le cas dans lequel le demandeur a explicitement retiré sa demande et le cas dans lequel l'autorité responsable de la détermination a rejeté une demande à la suite d'un retrait implicite, conformément à l'article 28 , $\$ 1$. 
décision prise dans une langue comprise ${ }^{40}$. Le droit à un entretien individuel est exigé par la directive "Procédures » dans tous les cas, à l'exception de l'examen préliminaire portant sur la recevabilité ${ }^{41}$, du cas dans lequel l'autorité entend prendre une décision positive ou si le demandeur n'est pas en état d'être entendu en raison de circonstances durables indépendantes de sa volonté ${ }^{42}$. Avant la réforme, l'OFPRA pouvait se dispenser de cet entretien soit lorsque le demandeur avait la nationalité d'un pays sûr, soit lorsqu'il était estimé que la demande était manifestement infondée ${ }^{43}$, hypothèse s'appliquant systématiquement aux demandes déposées en rétention administrative. Pour le Conseil constitutionnel, l'absence d'entretien ne faisant pas obstacle à un examen particulier des éléments produits ne portait pas atteinte à l'exercice du droit d'asile, cette dispense n'étant par ailleurs qu'une faculté à laquelle, au cas par cas, l'Office pouvait renoncer $^{44}$. Le projet de loi relatif à la réforme de l'asile a donc intégré cette garantie fondamentale «dans tous les cas» de procédure accélérée ${ }^{45}$.

Enfin, le droit à l'assistance juridique gratuite est maintenu dans tous les cas en procédure de recours ${ }^{46}$. On peut toutefois craindre que la mise en œuvre de l'exception visée au paragraphe 3 de l'article 20 , qui subordonne le droit à cette assistance à une "perspective tangible de succès », neutralise en partie l'exercice de ce droit. En effet, les motifs mêmes pour lesquels une procédure accélérée est mise en œuvre se rattachent directement aux perspectives d'obtenir une protection. Pays d'origine sûr, absence d'éléments pertinents, demandes manifestement infondées, demandes frauduleuses, autant de qualifications qui sont de nature à présumer l'absence de perspective tangible de succès. La procédure de contestation du refus d'aide juridique ne semble pas non plus compatible avec l'exigence de célérité de la procédure, puisque les États membres doivent prévoir un recours effectif contre la décision de refus d'octroyer cette aide lorsque celle-ci est prise par une autorité qui n'est pas juridictionnelle ${ }^{47}$. Pourtant, l'assistance juridique est déterminante dans l'exercice du droit au recours effectif. Quelles que soient les qualités du recours offert, il perd de son effectivité si, en fait, il n'est pas disponible. L'assistance juridique gratuite constitue, à cet égard, le corollaire de la disponibilité.

\section{Le contrôle juridictionnel des procédures accélérées}

Le contrôle juridictionnel des procédures accélérées constitue l'ultime volet des garanties essentielles de la procédure d'asile. Dans toutes les procédures relevant du droit de l'Union, un droit à un recours effectif est exigé $^{48}$. Cela implique que le recours soit disponible et utile en fait et suspensif en droit.

La décision par laquelle l'autorité responsable décide de la mise en ouvre de la procédure accélérée doit être susceptible d'être contestée devant une juridiction ${ }^{49}$. La directive «Procédures» n'apporte pas plus de précision, laissant aux États une totale autonomie procédurale. La question est de savoir si le contrôle de légalité exercé sur la décision plaçant la demande sous le régime de la procédure accélérée relève de la compétence du juge ayant à statuer sur le bien-fondé de celle-ci. Dans l'affaire Samba Diouf, la CJUE a considéré que l'article 39 de la directive $n^{\circ}$ 2005/85/CE du Conseil n'impliquait pas de prévoir un recours autonome contre la décision prise par l'autorité compétente pour statuer sur le régime de la procédure

[...] dès lors que les motifs qui ont conduit cette autorité à examiner le bien-fondé de ladite demande [...] peuvent être effectivement soumis à un contrôle juridictionnel dans le cadre du recours dont la décision finale de rejet est susceptible de faire l'objet ${ }^{50}$.

Interprétant une directive posant des normes minimales, la Cour s'est bornée à énoncer une conception minimale du droit au recours effectif. Or, on peut s'interroger sur la pertinence de la fusion des contrôles portant sur les motifs de la procédure accélérée et sur le bien-fondé de la demande d'asile. En effet, le but de la contestation de la procédure accélérée est de permettre au demandeur de jouir de la procédure dite normale, quant aux délais et quant aux garanties procédurales. Or, dans le cas d'une compétence unifiée, quelle peut être la conséquence de la reconnaissance d'une illégalité du placement en procédure accélérée? Si le juge annule la décision finale portant sur la protection internationale, l'intéressé se voit accorder le bénéfice de cette protection et, par voie de conséquence, bascule sous le régime de celle-ci. En cas de rejet de sa demande au fond, la reconnaissance de l'illégalité de la procédure accélérée

40. Art. 12 de la directive $2013 / 32 /$ UE.

41. Art. $42, \$ 2$, sous b, de la directive $2013 / 32 / \mathrm{UE}$

42. Art. $14, \$ 2$, sous a et b, de la directive $2013 / 32 / \mathrm{UE}$.

43. Art. L. 723-3 du CESEDA.

44. CC, 4 décembre 2003, $\mathrm{n}^{\circ}$ 2003-485 DC

45. Projet de loi relatif à la réforme de l'asile, art. 7 .

46. Art. 20 de la directive $2013 / 32 /$ UE.

47. Ibid., $\$ 3$.

48. Art. 47 de la Charte des droits fondamentaux de l'Union européenne.

49. Art. 46, $\$ 4$, de la directive 2013/32/UE.

50. CJUE, $2^{\mathrm{e}}$ ch., 28 juillet 2011, Brahim Samba Diouf c. ministre du Travail, de l'Emploi et de l'Immigration, pt 70. 
n'aura aucune conséquence concrète puisque la décision juridictionnelle aura mis fin à la procédure de protection ${ }^{51}$. Dans ces conditions, si la Cour a estimé qu'il

[...] ne serait pas compatible avec le droit de l'Union qu'une réglementation nationale telle que celle résultant de l'article 20, paragraphe 5, de la loi de 2006 puisse être interprétée en ce sens que les motifs qui ont conduit l'autorité administrative compétente à examiner la demande d'asile dans le cadre d'une procédure accélérée ne puissent faire l'objet d'aucun contrôle juridictionnel ${ }^{52}$ [et que] afin que l'exercice de ce droit soit effectif, il faut que le juge national puisse vérifier le bien-fondé des motifs qui ont conduit l'autorité administrative compétente à considérer la demande de protection internationale comme infondée ou abusive, sans que ceux-ci bénéficient d'une présomption irréfragable de légalités ${ }^{3}$.

En fait, ce contrôle juridictionnel bien effectif est dénué de toute portée.

En droit français, le contrôle de légalité des décisions plaçant les demandes en procédure prioritaire relève de la compétence du tribunal administratif en raison de la compétence du préfet pour les édicter. Faute de précision sur ce point de la directive, le projet de loi relatif à la réforme de l'asile a totalement fait sienne l'interprétation de la Cour. L'article 7 du texte soumis à l'examen de l'Assemblée nationale précise que

La décision de l'autorité administrative visée au III ne peut pas faire l'objet, devant les juridictions administratives de droit commun, d'un recours distinct du recours qui peut être formé, en application des article L. 731-1 et suivants, devant la Cour nationale du droit d'asile à l'encontre de la décision de l'office rejetant la demande ${ }^{54}$.

Autrement dit, le contrôle de légalité de la procédure et le fond de la demande ne peuvent faire l'objet que d'un recours unique. Ce n'est que dans le cas d'un placement en rétention administrative que le tribunal administratif retrouve cette compétence pour statuer sur la légalité de la procédure accélérée ${ }^{55}$.

\section{Le droit à un recours suspensif}

Le maintien sur le territoire durant l'examen du recours contre la décision initiale, en tant qu'il participe pleinement de l'effectivité du recours, est de plein droit ${ }^{56}$. En revanche, lorsque la demande est examinée en procédure accélérée pour les motifs visés à l'article $31, \$ 8$, de la directive, à l'exception du cas de l'introduction tardive d'une demande après une entrée ou un séjour irrégulier prolongé, le bénéfice du caractère suspensif doit être accordé par un juge. L'article $46, \$ 6$, précise en effet que cette décision revient à une juridiction qui, de sa propre initiative ou à la demande de l'intéressé, l'ordonne dans l'hypothèse où la décision rendue par l'autorité responsable mettrait fin au droit au séjour provisoire et que l'exercice d'un recours ne serait pas, par lui-même, suspensif. Le $\$ 8$ de l'article 46 impose aux États membres de conférer un effet suspensif à cette procédure particulière.

Le législateur européen a donc trouvé un compromis entre, d'une part, la procédure normale qui confère un caractère suspensif au recours contre la décision de l'autorité responsable et, d'autre part, le refus d'octroyer un caractère suspensif aux procédures accélérées, ce qui en fait leur spécificité.

Selon la Cour européenne des droits de l'homme, pour être effectif, un recours n'a pas à être nécessairement assorti d'un caractère suspensif. Ce n'est que dans le cas où le grief porte sur un risque d'être exposé à un traitement prohibé par l'article 3 de la Convention et compte tenu du caractère irréversible du dommage susceptible d'être causé que ce caractère doit être prévu ${ }^{57}$. Les motifs justifiant l'examen d'une demande de protection internationale se rapportent, plus ou moins indirectement, à des risques d'être soumis à des traitements prohibés par la Convention, que ce soit au regard des stipulations précitées ou du droit à la vie ou à la liberté et à la sûreté. S'agissant plus particulièrement du contentieux de l'asile, la Cour européenne des droits de l'homme a estimé que, dans l'affaire qui lui était soumise, le défaut de recours suspensif en procédure prioritaire était de nature à affecter le caractère effectif du recours disponible ${ }^{58}$. Tel est également l'exigence posée par la Cour de justice de l'Union européenne qui, dans l'affaire Abdida, a considéré que «[...] la gravité et le caractère irréparable du préjudice résultant de l'éloignement d'un ressortissant de pays tiers vers un pays dans lequel il existe un risque sérieux qu'il soit soumis à des traitements inhumains ou dégradants $»^{59}$ imposent de prévoir un recours suspensif.

51. On peut réserver l'hypothèse dans laquelle le Conseil d'État a jugé que la violation d'une garantie substantielle autorisait la juridiction compétente à statuer sur le fond pour annuler la décision litigieuse et renvoyer la demande devant l'autorité responsable pour être réexaminée (CE, 10 octobre 2013, Office français pour la protection des réfugiés et des apatrides, $\mathrm{n}^{\circ} 362798$ ).

52. CE, 10 octobre 2013, Office français pour la protection des réfugiés et des apatrides, $\$ 58$.

53. Ibid., $\$ 61$.

54. Projet de loi relatif à la réforme de l'asile, art. 7 .

55. Voir infra.

56. Art. $46, \S 5$, de la directive $2013 / 32 / \mathrm{UE}$

57. Cour EDH, 26 avril 2007, Gebremedhin c. France, $\mathrm{n}^{\circ} 25389 / 05$.

58. Ibid., $\$ 156$.

59. CJUE, GC, 18 décembre 2014, Centre public d'action sociale d'Ottignies-Louvain-la-Neuve c. Moussa Abdida, aff. C-562/13, pt 50. En l'espèce, l'affaire portait sur le recours dirigé contre une décision de retour concernant un ressortissant d'État tiers dont l'état de santé pouvait s'opposer à l'exécution de son éloignement. La solution retenue par la Cour, qui s'inspire directement et expressément de la jurisprudence de la Cour de Strasbourg, serait alors parfaitement transposable au contentieux du droit au séjour provisoire des demandeurs d'asile placés en procédure accélérée. 
En droit français, avant la réforme du droit d'asile de 2015, le placement en procédure prioritaire privait le demandeur du caractère suspensif du recours introduit devant la CNDA. Bien que la procédure soit en partie écrite, elle comprend une phase où l'oralité occupe une place essentielle. Dans un contentieux où les requérants sont souvent dépourvus de preuve matérielle appuyant leurs allégations de craintes de persécution, l'audience au cours de laquelle le demandeur pourra exposer avec plus de précision la nature de ses craintes et surtout répondre aux questions susceptibles d'être posées par les membres de la Cour est déterminante.

Le projet de loi relatif à la réforme de l'asile a donc intégré un mécanisme de recours suspensif lorsque la demande de protection a été menée en procédure accélérée, avec la spécificité d'un examen en formation de juge unique qui aura à statuer dans un délai d'un mois ${ }^{60}$. Tous les demandeurs d'asile, quel que soit le régime sous lequel est placé l'examen de leur demande, devraient bénéficier $\mathrm{du}$ droit de se maintenir sur le territoire ${ }^{61}$. Toutefois, le législateur a prévu quelques exceptions à cette généralisation qui, bien qu'elles ne soient pas nombreuses, couvrent en réalité d'un point de vue quantitatif la majorité des cas d'ouverture de procédures accélérées. Ainsi, il est prévu que le droit de se maintenir sur le territoire prend fin soit après une décision d'irrecevabilité de l'OFPRA ${ }^{62}$ ou lorsque la demande de réexamen vise à faire échec à une mesure d'éloignement prononcée ou imminente ${ }^{63}$. Nous savons qu'avant la réforme de l'asile, notamment en 2012, sur les 14800 procédures prioritaires, 6855 d'entre elles étaient fondées sur la circonstance que le demandeur était originaire d'un pays d'origine sûr ${ }^{64}$. Ce qui révèle que près des 8 ooo procédures restantes devaient être fondées sur le $4^{\circ}$ de l'article L. 741-4 du CESEDA concernant les demandes frauduleuses, dilatoires ou ayant pour but de faire échec à une mesure d'éloignement, la part des procédures prioritaires fondées sur la sauvegarde de l'ordre public étant infinitésimale. Il faut préciser que toute procédure d'asile qui se conclut par un refus conduit à l'édiction d'une mesure d'éloignement. De ce fait, bien que ce ne soit pas expressément formulé de cette façon, toute demande de réexamen d'une demande de protection risque de relever de cette disposition privant le demandeur du droit de rester sur le territoire. En effet, soit la demande de réexamen est irrecevable, soit la demande de réexamen est recevable mais, parce qu'il s'agit d'un réexamen, elle fait probablement suite à une mesure d'éloignement, cas dans lequel la demande pourra être regardée comme ayant pour but d'y faire obstacle. Ainsi, bien que proclamant la généralisation du droit de rester sur le territoire lorsque les demandes sont placées en procédure accélérée, en fait, la plupart de celles-ci risquent d'être soustraites au bénéfice de ce droit.

Enfin, si l'intéressé est placé en rétention administrative et dépose sa demande de protection à cette occasion, il ne pourra bénéficier du caractère suspensif du recours que sur demande introduite auprès du magistrat désigné du tribunal administratif, dans les 48 heures suivant la notification de la décision de l'OFPRA, qui aura la charge de vérifier si la demande d'asile n'a pas été déposée dans le seul but de faire échec à l'exécution de la mesure d'éloignement ${ }^{65}$. Autrement dit, le caractère suspensif et donc effectif du recours devant la CNDA dépendra d'une part, d'une demande expresse du demandeur d'asile qui devra introduire une requête en français et motivée auprès de la juridiction administrative et, d'autre part, de la décision d'un magistrat qui, en toute hypothèse, aura déjà été amené à statuer sur le recours dirigé contre la mesure d'éloignement en le rejetant. Le système ainsi mis en place semble aussi inefficace, du point de vue de la garantie des droits, que celui qui fût critiqué par la Cour européenne des droits de l'homme dans l'affaire I. M. c. France. Inefficace dans la mesure où le requérant se heurtera aux mêmes difficultés pour établir le bienfondé de ses prétentions compte tenu des conditions dans lesquelles il est amené à exercer ses droits. Mais surtout, si le placement en procédure accélérée devrait théoriquement perdre son caractère automatique, il est à peu près certain que les demandes d'octroi d'un caractère suspensif seront dans leur grande majorité rejetées au motif que les demandes d'asile n'auraient que pour but de faire échec à l'exécution de la mesure d'éloignement. Indépendamment de la complexité décourageante du système ainsi mis en place, il paraît improbable qu'il permette au demandeur, dans un nombre significatif de cas, de se maintenir sur le territoire dans l'attente de l'examen de son recours. C'est ainsi que l'exercice de transposition de la directive «Procédures» semble réussir à concilier deux exigences contradictoires : l'apparence du respect du droit de l'Union et le maintien des pratiques antérieures. En France, sur ce point au moins, la directive "Procédures» n'aurait donc changé que les textes sans changer le droit, dans le sens du droit qui s'applique, le droit qui est à l'œuvre.

Si, comme l'a rappelé la Cour européenne des droits de l'homme, les procédures accélérées répondent à :

[...] la nécessité pour les États confrontés à un grand nombre de demandeurs d'asile de disposer des moyens nécessaires pour faire face à un tel contentieux [...] [et] que les procédures d'asile accélérées, dont se sont dotés de

60. Projet de loi relatif à la réforme de l'asile, art. 14 .

61. Ibid., art. 10

62. Décision prise soit lorsque le demandeur bénéficie d'une protection accordée par un autre État membre de l'UE, d'une protection dans un autre État tiers et qu'il y est réadmissible ou en cas de réexamen sans assortir cette demande d'éléments nouveaux.

63. Projet de loi relatif à la réforme de l'asile, art. 14.

64. Les étrangers en France, p. 107.

65. Projet de loi relatif à la réforme de l'asile, art. 9. 
nombreux États européens, puissent faciliter le traitement des demandes clairement abusives ou manifestement infondées ${ }^{66}$

Elles constituent aussi et surtout un moyen d'appréhender les demandes de protection internationale au prisme de la lutte conte l'immigration irrégulière, justifiant ainsi les mesures dérogatoires qui, supposées respecter les garanties fondamentales, en constituent des aménagements qui en affectent leur substance. Cet amalgame entre immigration irrégulière et demande de protection internationale semble désormais bien ancré dans l'esprit des institutions, comme le démontre l'arrêt du Conseil d'État du 18 juin 2014 dans lequel la haute juridiction associe les ressortissants syriens fuyant leurs pays en raison du climat de violence généralisé à des migrants clandestins ${ }^{67}$. Les procédures accélérées devraient être marginales. Pourtant, la pratique démontre que la plasticité conceptuelle permet d'y recourir de manière systématique dans des circonstances telles que la rétention administrative.

Si la directive «Procédures» conduit à une élévation du standard des garanties dans la procédure d'asile dans les États membres de l'Union, les dispositions relatives aux procédures accélérées résultent d'un mauvais compromis grâce auquel les États maintiennent pleinement leur marge de manœuvre répressive.

Le 30 avril 2015.

66. Cour EDH, 2 février 2012, I. M. c. France, $\$ 142$

67. CE, 18 juin 2014, Anafé et Gisti, $\mathrm{n}^{\circ} 366307$. 